\title{
Geste individuel, mémoire collective. Le jeu du pan dans les steelbands de Trinidad \& Tobago
}

\section{Aurélie Helmlinger}

\section{(2) OpenEdition}

Journals

Édition électronique

URL : http://journals.openedition.org/ethnomusicologie/1836

ISSN : 2235-7688

Éditeur

ADEM - Ateliers d'ethnomusicologie

Édition imprimée

Date de publication : 1 décembre 2001

Pagination : 181-202

ISBN : 2-8257-07-61-9

ISSN : 1662-372X

\section{Référence électronique}

Aurélie Helmlinger, «Geste individuel, mémoire collective. Le jeu du pan dans les steelbands de Trinidad \& Tobago », Cahiers d'ethnomusicologie [En ligne], 14 | 2001, mis en ligne le 04 avril 2012, consulté le 21 avril 2019. URL : http://journals.openedition.org/ethnomusicologie/1836

Ce document a été généré automatiquement le 21 avril 2019

Tous droits réservés 


\title{
Geste individuel, mémoire collective. Le jeu du pan dans les steelbands de Trinidad \& Tobago
}

\author{
Aurélie Helmlinger
}

1 En visitant Trinidad, il est impossible d'ignorer longtemps l'existence du pan ${ }^{1}$. On ne tarde pas à découvrir cet idiophone mélodique (appelé aussi steeldrum ou steelpan) fabriqué par catachrèse ${ }^{2}$ de bidons de pétrole. Après l'avoir embouti à l'aide d'une masse, on façonne sur sa surface devenue concave un certain nombre de facettes dont l'accordage sur une gamme occidentale tempérée sera facilité par une cuisson préalable, sur un feu nourri des résidus d'hydrocarbures. Le facteur (appelé tuner) martèle chaque note, séparée de la voisine par une rainure, pour lui donner une forme légèrement convexe, ajustant ainsi la note fondamentale. S'il a une bonne oreille et en s'aidant parfois d'appareils électroniques, il peut également accorder les partiels sur le modèle des premières notes de la série harmonique, donnant à l'instrument un timbre brillant, qu'un éventuel chromage par électrolyse renforcera et maintiendra plus longtemps. Ainsi fabrique-t-on aujourd'hui cet instrument dont il existe de nombreux types de tessitures différentes, reconnaissables par le nombre de bidons et la taille de leur jupe.

Inventé à la fin des années trente par la population d'origine africaine ${ }^{3}$ dans les quartiers défavorisés de Port-of-Spain, le pan a pris progressivement place dans le carnaval. Suite à l'habitude d'ajouter aux tambours traditionnels, puis aux bambous percutés et pilonnés (les tamboo-bamboo), des instruments de récupération métalliques, il est le fruit de découvertes acoustiques dues aux propriétés sonores de sa matière vibrante. D'abord très mal considéré, il est peu à peu accepté puis érigé en symbole culturel de Trinidad par des intellectuels nationalistes qui prennent parti en sa faveur. A l'indépendance (1962), le PNM - parti nationaliste au pouvoir - favorisera son développement par de nombreuses décisions politiques comme la création de compétitions nationales.

3 "Seul instrument de musique inventé au $\mathrm{XX}^{\mathrm{e}}$ siècle", clament un peu présomptueusement les prospectus touristiques. Et le pays entier semble fêter chaque 
année, au moment du carnaval, son instrument national en une gigantesque compétition appelée Panorama, où d'immenses groupes d'une centaine de personnes, les steelbands, s'affrontent par la performance d'un morceau de dix minutes aux arrangements virtuoses. Le soir de la finale (après l'épreuve des préliminaires et des demi-finales), le pays retient son souffle, et ceux qui ne peuvent assister directement au spectacle le suivront probablement à la radio ou à la télévision, pour infirmer ou confirmer les fiévreux pronostics qui fusent depuis le début des répétitions quotidiennes, plus d'un mois auparavant.

4 C'est au sein de ces compétitions de steelbands que le geste musical assume un rôle outrepassant ce qui ne serait que l'exécution d'un matériau musical construit indépendamment. Du point de vue individuel d'abord, le geste, guidé par la configuration des notes sur l'instrument, peut avoir des conséquences sur les choix musicaux de l'arrangeur. Collectivement ensuite, un certain nombre de constatations semblent suggérer que quelque chose d'important se joue entre les gestes simultanés des musiciens et la mémorisation même du répertoire ${ }^{4}$.

\section{La musique des steelbands}

5 Depuis l'indépendance, en 1962, l'activité des steelbands est largement orchestrée par une association nationale, "Pan Trinbago", financée par le gouvernement. Différentes compétitions rythment la vie musicale au fil des mois, toujours ouvertes par l'hymne national, écouté par tous avec grand respect. Par leurs règles elles dessinent le paysage musical : à chaque concours correspond un type de pièce et un nombre approximatif de musiciens.

6 Le Panorama, dont la finale a lieu le samedi soir précédant le carnaval, est l'événement le plus mobilisateur ${ }^{5}$. La pièce doit impérativement être l'arrangement d'un calypso diffusé sur les ondes radio de Trinidad dans l'année précédente. Le calypso, chanson de satire sociale à l'harmonie tonale et accompagnée d'un rythme caractéristique à deux temps, a été rapidement intégré dans le répertoire des steelbands. Les règles du Panorama ont par la suite donné naissance à un nouveau type, destiné à fournir le matériau musical de ces pièces de compétition. Un tel calypso, réalisé à grands renforts de synthétiseurs et de boîtes à rythmes, plus que par son impertinence textuelle, fera une brillante carrière s'il passe souvent à la radio et est repris par le « Panorama tune » vainqueur de la compétition.

7 Plusieurs steelbands peuvent donc jouer un même thème, puisque ces calypsos sont une sorte de répertoire annuel auquel chacun puise. Mais les différents arrangements, inspirés de la musique symphonique occidentale, signeront leur originalité : l'arrangeur du steelband compose ses propres introductions, développements et conclusions, devant impressionner les juges et enthousiasmer le public. Très suivi par la population, retransmis en direct à la radio et à la télévision avec force commentaires passionnés, le Panorama est sans conteste l'événement phare de la vie musicale des steelbands.

8 Au mois d'octobre, le répertoire adopte un autre style avec la compétition du Festival, où l'usage veut que les groupes, d'environ 40 à 60 musiciens, reprennent à nouveau des calypsos mais aussi des pièces de musique classique occidentale. Lors de ces compétitions et pour les fêtes privées qu'animent occasionnellement les steelbands, le répertoire, appris par cœur, ne connaît pratiquement pas de variation. Seule une compétition, «Pan 
Ramajay $^{6}$ ", juge l'improvisation dans un style inspiré du jazz, de formations de moins de dix personnes.

\section{Geste et choix musicaux}

9 L'étude du geste musical constitue une récente voie de recherche de l'ethnomusicologie, son domaine pouvant dépasser le simple cadre de l'exécution matérielle d'une pensée musicale abstraitement préconçue. Outre le rôle que le geste pourrait avoir dans l'attribution de représentations sur les musiciens, son étude intéresse la discipline pour son rôle dans la cognition musicale. Les contraintes physiques auxquelles l'instrumentiste est confronté participent à la forme de la musique : les caractéristiques techniques du jeu d'un instrument donnent nécessairement lieu à des combinaisons faciles, difficiles ou impossibles. Un accord de piano sera par exemple forcément soumis à l'étendue maximale couverte par chacune des deux mains du musicien sur le clavier. L'un des premiers à avoir abordé ce domaine de recherche, John Baily $(1977,1987)$ a ainsi analysé des formules musicales de luths d'Afghanistan à la lumière de caractéristiques motrices de leur exécution. Loin de vouloir minimiser le contexte culturel qui façonne l'esthétique générale d'une musique, cette approche permet de mieux cerner les choix de l'instrumentiste, et de comprendre la récurrence de certains traits par les regards croisés des contraintes gestuelles et culturelles.

10 La musique des steelbands, comme on l'a vu, laisse peu de place à l'improvisation, mais l'extrême rapidité des pièces de compétition fournit une contrainte culturelle qui peut amener les arrangeurs, tous pannistes ${ }^{7}$ eux-mêmes, à composer en tenant compte des impératifs gestuels. L'un d'entre eux, Jit Samaroo, m'a ainsi déclaré renoncer parfois à certaines idées de composition du fait de leur trop grande difficulté, en ajoutant «Je connais mes musiciens, je sais ce qu'ils sont capables de jouer ». La contrainte gestuelle, dans ce type d'orchestre, pourrait être délicate puisqu'il existe dans un steelband au moins une dizaine de types de steeldrums aux topologies très différentes les unes des autres, et corollairement aux caractéristiques gestuelles diverses. Mais la plupart des instruments fournissent la grille harmonique sur un ostinato rythmique ; la partie qu'ils exécutent est donc plutôt guidée par la grille du calypso que par la gestuelle qu'ils impliquent.

11 En revanche, du fait de la rapidité d'exécution, la partie mélodique composée par les arrangeurs (non issue de la reprise du calypso) est davantage susceptible de contenir des formules inspirées de la facilité de réalisation de certains mouvements. Les tenors assument cette fonction mélodique, généralement accompagnés une tierce en dessous par les double tenors, moins nombreux. Mais au cours des développements, les autres sections d'instruments sont amenées à exécuter tour à tour un passage mélodique, leur «solo", qui doit mettre en valeur leur virtuosité.

12 Le présent travail s'est concentré sur l'étude du tenor, constitué d'un seul bidon, en tant qu'instrument mélodique privilégié et à la configuration très répandue. Précisons que les steeldrums sont constitués de un à une dizaine de pans. Le placement des bidons va de l'horizontale à la verticale ${ }^{8}$. Pour le tenor et les instruments doubles (double tenor, double second, double guitar), les pans, suspendus à des montants métalliques, sont inclinés d'environ $25^{\circ}$ par rapport à l'horizontale. Les steeldrums plus graves se placent devant ou autour du musicien, certains pans se trouvant verticalement devant lui, le centre environ à hauteur des épaules. Les nine basses, par exemple, comportent six pans horizontaux (ou 
légèrement inclinés) et trois verticaux. Le fait que les bidons restent entiers pour servir de caisses de résonance rend les basses particulièrement imposantes (fig.1).

Fig. 1: Nine bass de Tokyo.

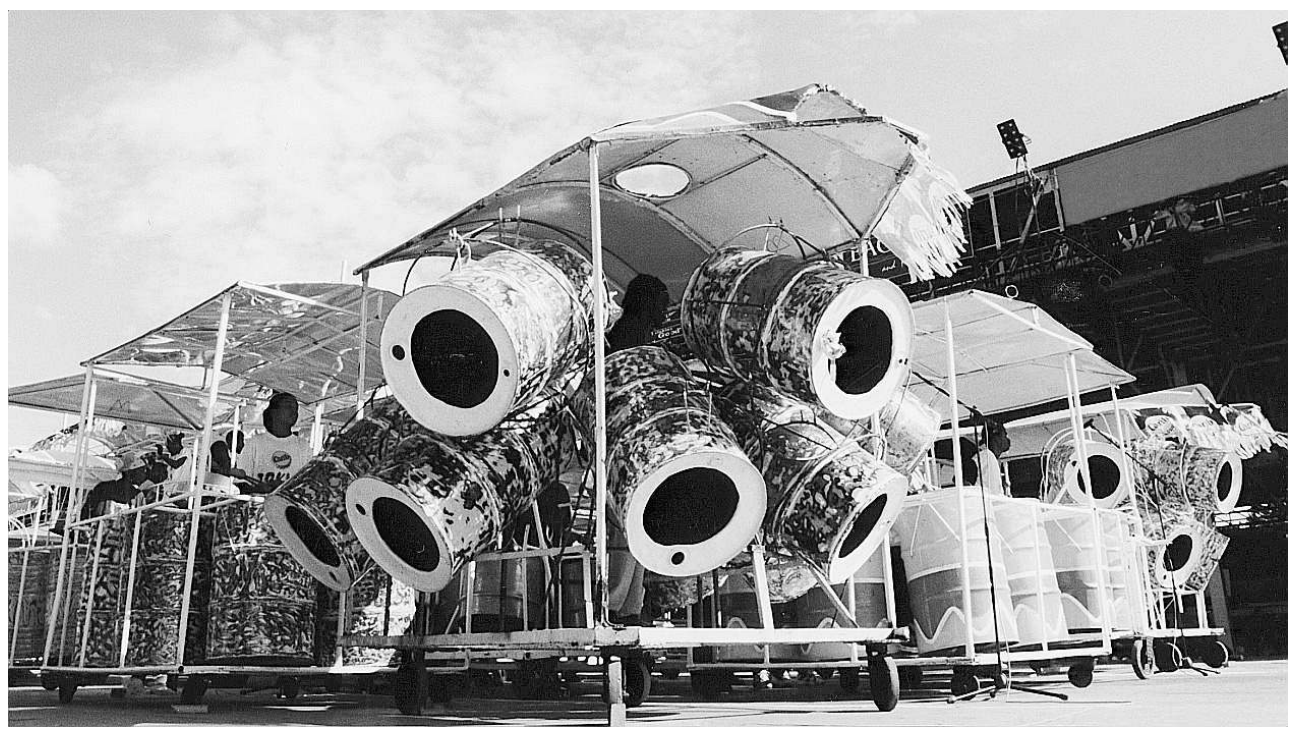

\section{Jeu du tenor, topologie}

Le joueur de tenor se tient toujours debout, le point le plus bas de l'axe de jeu se trouvant environ au niveau de l'aine. Dans cette posture, le buste droit et les mains plongées dans l'instrument, le panniste peut exécuter un certain nombre de mouvements: les bras et surtout les poignets se meuvent pour atteindre les notes avec les baguettes bien sûr, mais l'axe vertébral peut également basculer pour rapprocher le buste du pan, en particulier dans les passages difficiles (fig. $2 a$ et $2 b$ ). 
Fig. 2a-b : Joueur de tenor.

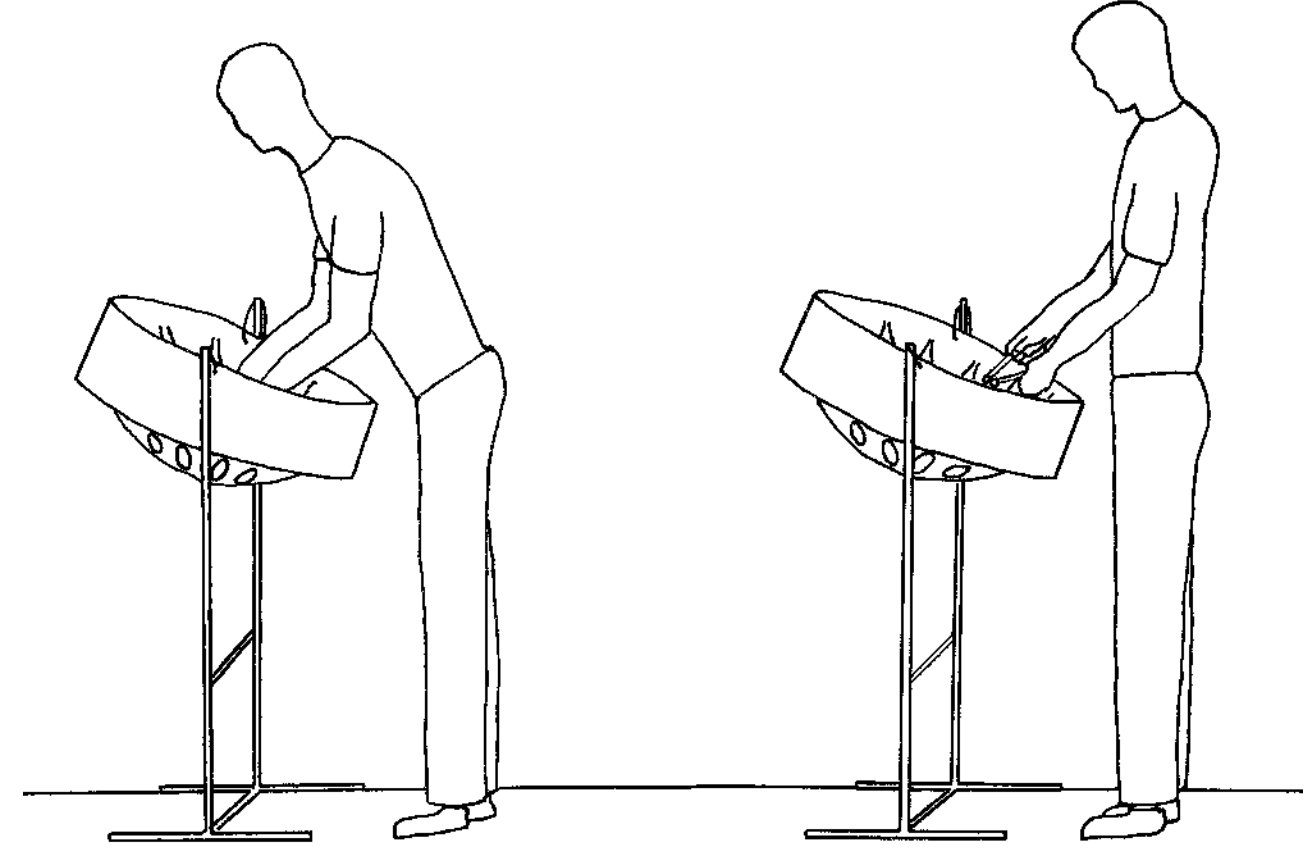

14 Une des principales difficultés gestuelles réside dans les doigtés, la répartition des notes de la mélodie entre la main droite et la main gauche. Et ceux-ci, même si les choix peuvent parfois différer selon les musiciens, sont directement liés à l'ordre de placement des notes sur l'instrument, la topologie du tenor (fig. 3).

Fig. 3 : Topologie du tenor.

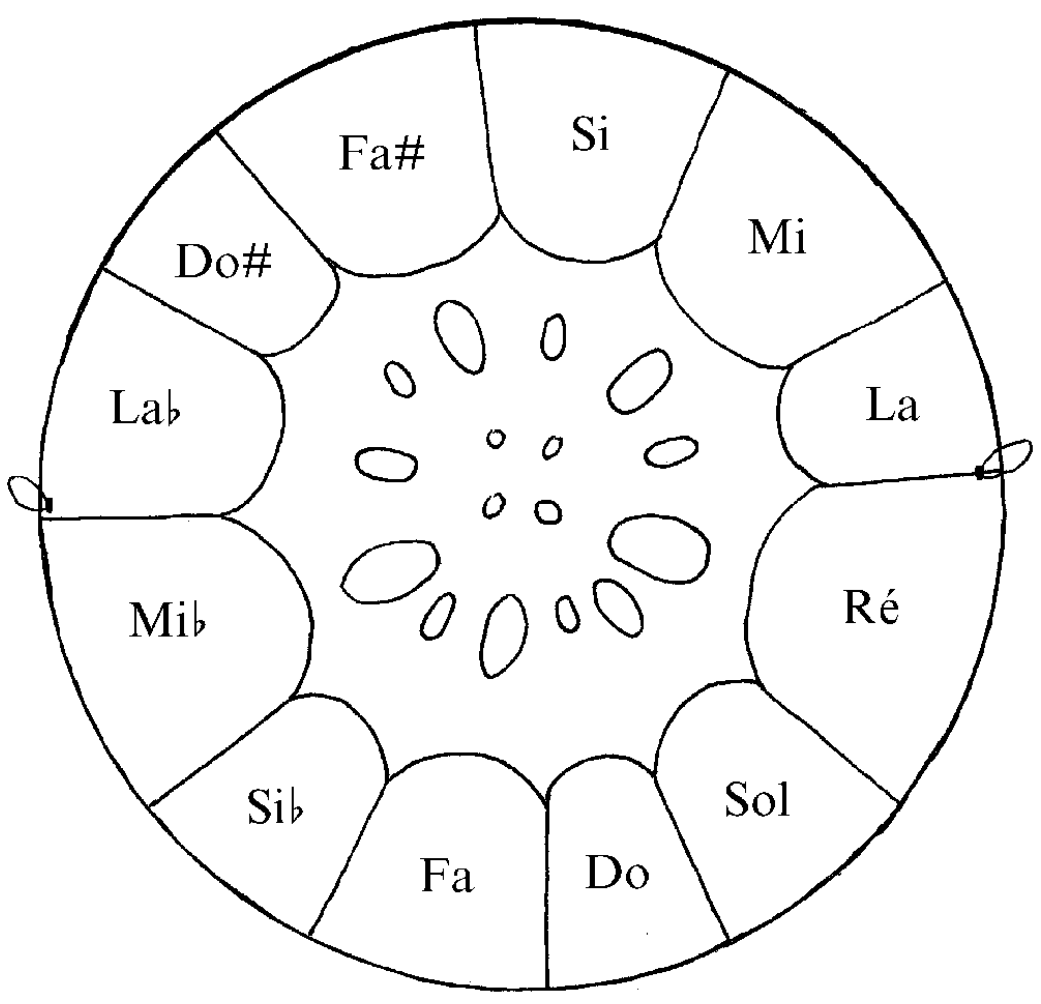


( constante, quelle que soit la tonalité (fig. 4a-b). Mais si cela facilite le stockage des informations, en économisant les figures à mémoriser, cela n'empêche que la forme des gammes diatoniques est visuellement et donc gestuellement assez irrégulière.

Fig. 4a-b : Gammes diatoniques majeure et mineure.
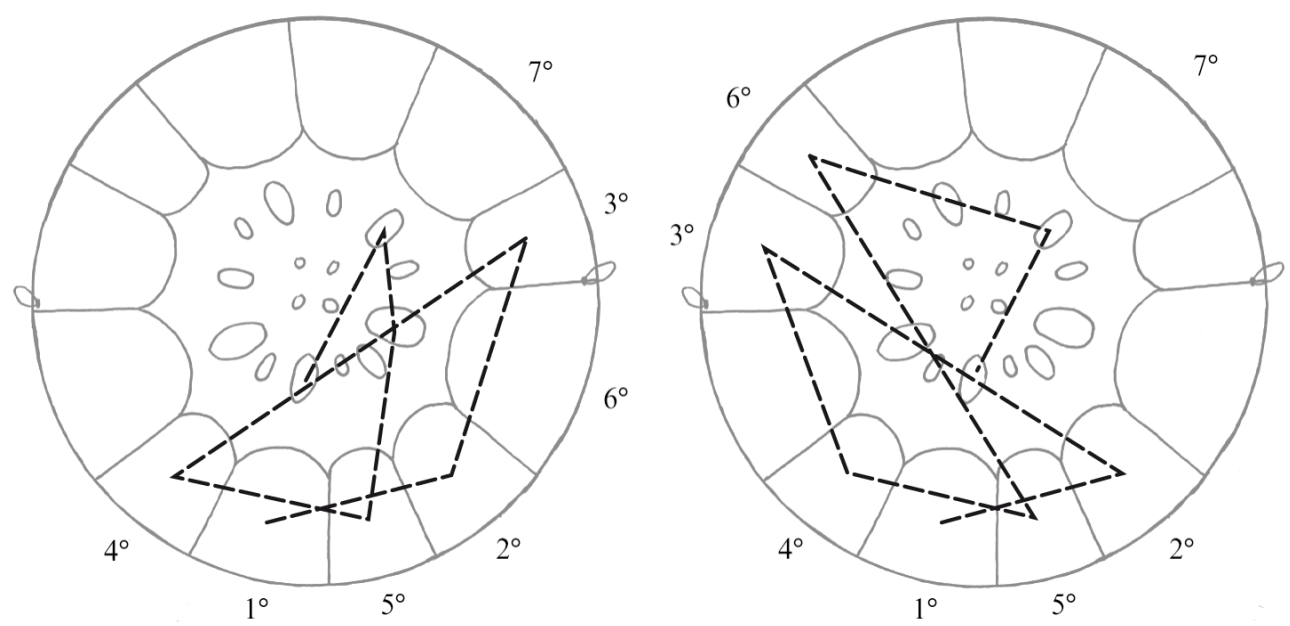

\section{Effets musicaux}

\section{Répétition d'une cellule ${ }^{9}$}

Le premier trait musical issu d'une préférence motrice se retrouve dans beaucoup d'arrangements dans ce que l'on appelle les runs. Il s'agit des passages où une longue série de doubles-croches est jouée sans interruption à grande vitesse, tout le monnayage étant marqué. Ils doivent être à la fois faciles à mémoriser et à exécuter. 
19 L'avantage principal de la répétition d'une cellule est de créer un mouvement moteur régulier, qui permet une exécution relativement automatique. La répartition entre les frappes de la main droite et celles de la main gauche acquiert un aspect mécanique absent du jeu habituel dans la gamme diatonique, à la répartition toujours irrégulière. Le geste se répète à l'identique sur un doigté de base comme l'alternance entre main droite et main gauche pour les cellules de quatre notes et deux notes doublées de la même main pour les cellules de trois notes (exemples fig. 5).

Fig. 5 : Transcription de répétition d'une cellule

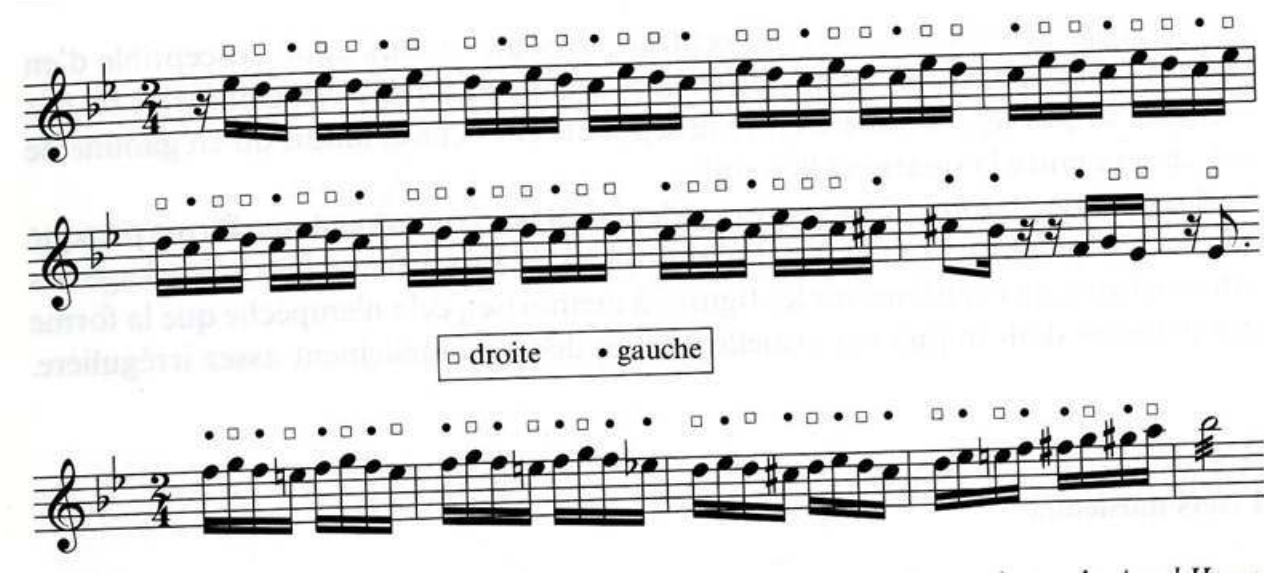

a. extrait de Get something and wave de Angel Harps, 1992, arrangement de Clarence Morris ; b. extrait de Pan Messiah de Merry Tones, 1998, arrangement de Kendall Lewis.

Ce trait comprend aussi nécessairement un nombre restreint de notes à retenir. Dans un run, lorsqu'aucun silence ne vient ponctuer une phrase, il est beaucoup plus difficile d'anticiper le geste et donc de le mémoriser. La vitesse est trop grande pour pouvoir entièrement compter sur la mémoire visuelle, et seuls les réflexes gestuels entrent en jeu. On comprendra qu'il est bien plus facile d'anticiper le jeu de trois ou quatre notes que l'on répète, que d'en mémoriser une dizaine de différentes. La répétition concerne le plus souvent des cellules de trois notes, qui ont en plus l'avantage de créer un décalage rythmique tout à fait au goût des Trinidadiens, en plaçant un accent sur les croches pointées.

\section{Les chromatismes}

21 La caractéristique probablement la plus marquante des morceaux de compétition est l'abondance des chromatismes mélodiques. Ce phénomène est présent dans l'ensemble du répertoire, tout au long des morceaux, très nettement perceptible y compris pour un auditeur non averti. Il est à ce point frappant qu'il se trouve à plusieurs reprises mentionné dans la thèse de Shannon Dudley (1997), à travers des citations d'informateurs considérant les chromatismes comme un effet contestable du Panorama :

Beverly Griffith : «Et quelquefois, on ne s'entraîne pas en rythme du tout, parce que le morceau est très compliqué, avec beaucoup de notes dans les runs, et vous entendez seulement bdddddup, bdddddup! (imitant le son d'une rapide montée chromatique) pendant environ une heure » (in Dudley $1997: 243)^{10}$. 

que j'écoute les cinq premiers groupes et réalise que tout ce que j'entend ce sont des chromatiques, d'inutiles chromatiques, je vais rentrer chez moi» (ibid.: 214). [...] «Par exemple, si vous remarquez, tous, la majorité des groupes, $90 \%$ des groupes jouent des chromatismes. La plupart des compositeurs, quand tu composes un morceau, tu ne mets pas seulement un chromatisme parce que les autres compositeurs l'ont fait. Tu arranges ton morceaux aux capacités que tu as et tu sais ce que tu veux entendre. Tu dois arranger le morceau pour toi-même et pour les joueurs, pas pour les juges. Parce que les juges veulent entendre des chromatismes. Tu ne peux pas et tu ne dois pas mettre des chromatismes seulement parce qu'ils disent que c'est le moyen de faire monter l'excitation - ce n'est pas le seul moyen de faire monter l'excitation » (ibid. : 244) ${ }^{11}$. Cesmoignages montrent clairement l'omniprésence du phénomène. Les exemples musicaux sont en effet innombrables. La raison évoquée par Shannon Dudley et ses informateurs est claire : l'usage intensif des chromatismes est dû à l'escalade de virtuosité qu'a entraînée la compétition. Pour gagner, il faut convaincre les juges de sa supériorité technique, impressionner la foule. Il s'agit d'une conséquence directe de la volonté de domination. Mais cette explication ne permet pas de comprendre totalement un phénomène à ce point présent. Les arrangeurs pourraient après tout choisir d'autres types de gammes (ils utilisent parfois les gammes diatoniques, mais de manière infiniment moins récurrente) pour démontrer leurs qualités techniques, ou même tout simplement n'importe quelle phrase, pourvu qu'elle soit longue et rapide.

Mais la gamme chromatique présente l'atout majeur de s'accompagner d'une gestuelle particulièrement facile sur le tenor, puisqu'elle se joue dans une alternance presque totale entre la main droite et la main gauche. La symétrie de la topologie place en effet systématiquement les intervalles par demi-ton presque en face les uns des autres. Le tout forme donc, comme on peut le voir dans la fig. 6 (où est mentionné le doigté12) une figure géométrique étoilée dont l'équilibre visuel facilite aussi la mémorisation. Son intérêt, comme pour la répétition de cellule, est d'offrir une régularité dans le mouvement, un équilibre moteur entre les frappes de la main gauche et celles de la main droite. 
Fig. 6 : Gamme chromatique.

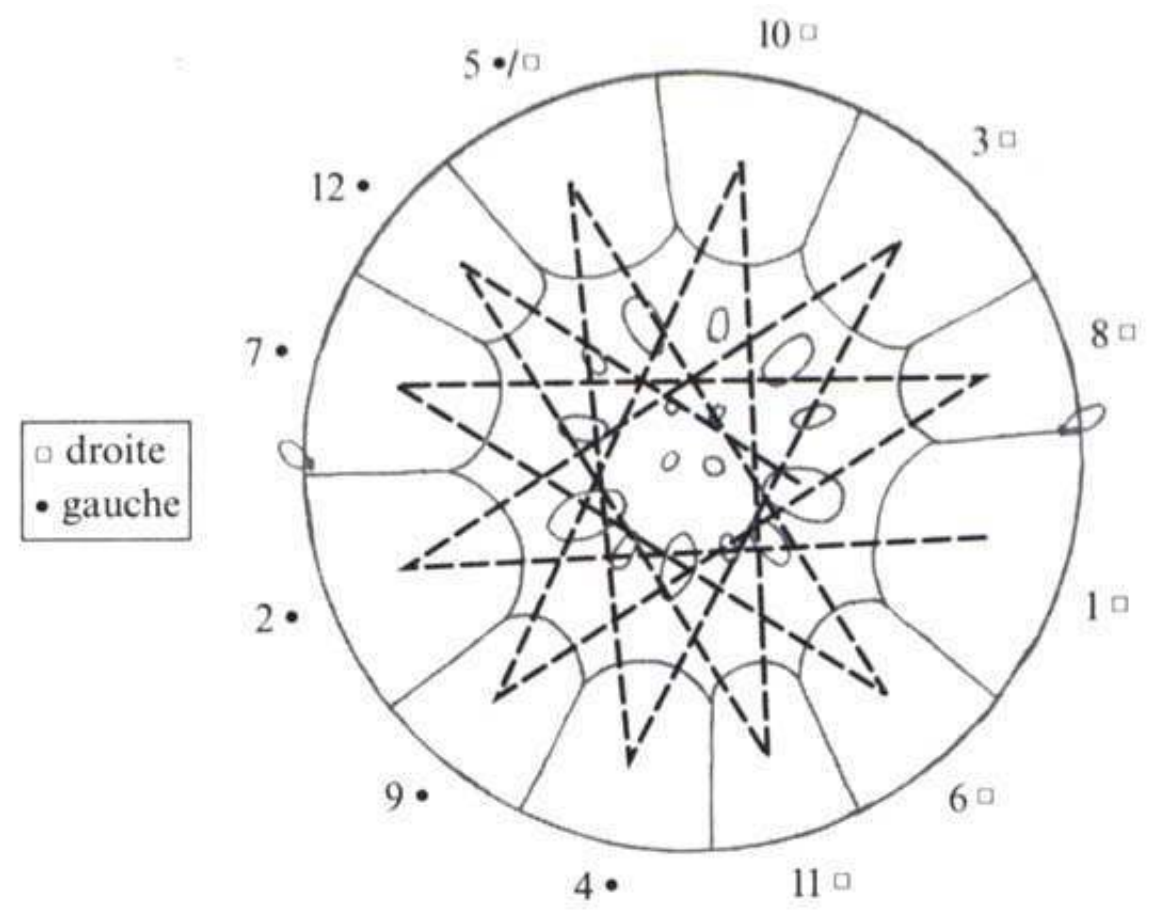

《Le pan, c'est facile... si tu répètes tes chromatiques " ${ }^{13}$, disait un jour Selwyn Henry, panniste à Pamberi. Il est clair que lorsqu'on a intégré visuellement et gestuellement cette gamme, de nombreux traits sont grandement facilités. On n'a plus qu'à retenir la note de départ, son placement rythmique, et on laisse ensuite aller ses mains jusqu'à celle d'arrivée. Les arrangeurs ont donc compris qu'ils pouvaient ainsi produire de longues phrases impressionnant les juges et le public, tout en ménageant relativement leurs musiciens. Les chromatismes mélodiques sont utilisés ainsi dans tous les passages composés par l'arrangeur, les plus longs se retrouvant systématiquement en fin d'introduction, et à la fin du morceau (fig. 7). 
Fig. 7 : Transcriptions de chromatismes mélodiques

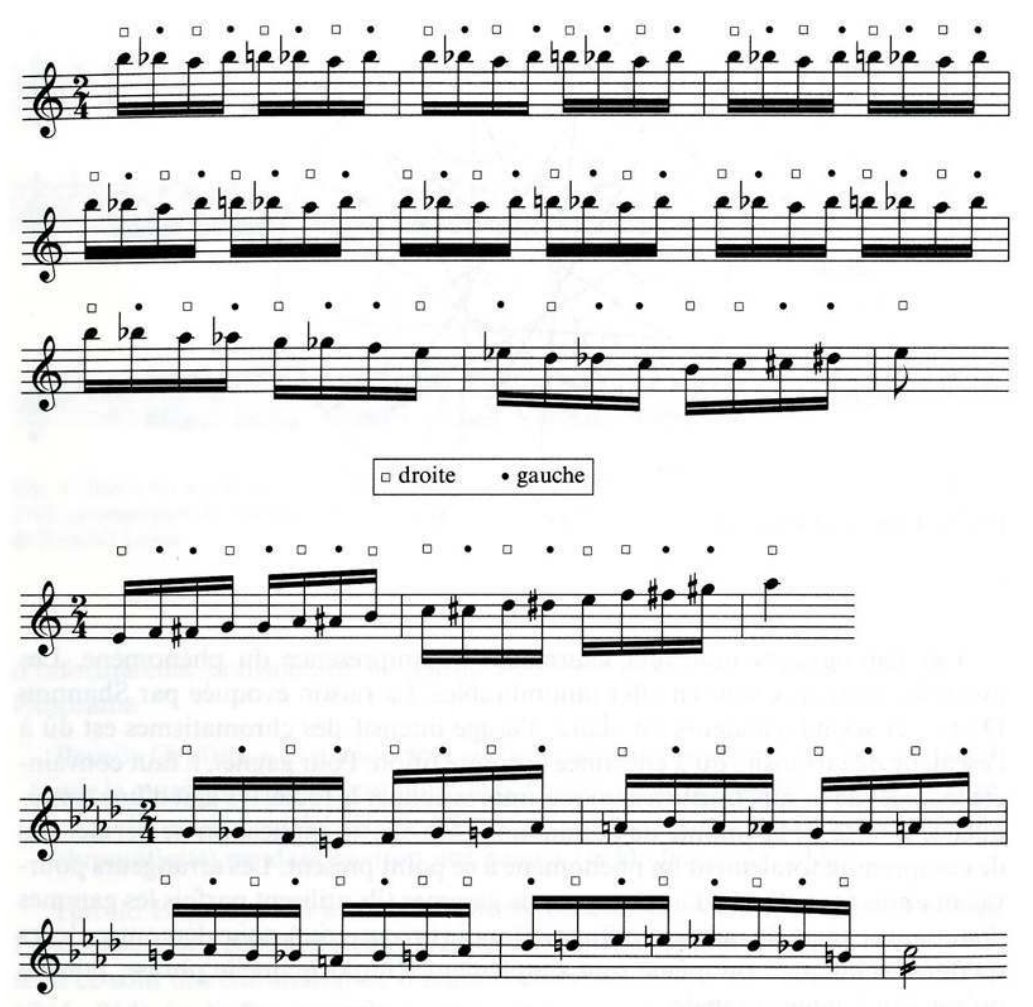

a) Extrait de Birthday Party par Phase II, 1996, arrangement de Len « Boogsie » Sharp.

b) Deux extraits de Pan in A Minor, par les Renagades, 1987, arrangement de Jit Samaroo.

Mais l'explication gestuelle ne doit pas occulter l'aspect esthétique qui joue aussi un rôle important. Car en plus de ses intérêts gestuels, visuels de et son effet sur les juges, la gamme chromatique crée un effet de montée vers une sorte d'explosion musicale qui sied bien à l'esprit du Panorama. La musique de cette compétition doit être une sorte d'éruption de joie, comme l'est d'ailleurs l'ensemble du carnaval. Si des chromatismes apparaissent tout au long de l'arrangement, les longues montées précèdent toujours les moments forts du morceau : l'arrivée du thème du calypso (que tout le monde connaît par chœur pour l'entendre depuis des semaines sur toutes les radios) et les accords de la coda. Peut-être pourrait-on associer cet effet musical avec le sentiment de communion nationale mis en valeur dans la thèse de Steven Stuempfle : "J'ai discuté précédemment de certains messages que véhicule le steelband concernant l'ethnicité, les différences de classes et intégration. Pendant les performances, cependant, d'autres dimensions prévalent. En écoutant les groupes s'efforcer d'exécuter de complexes arrangements musicaux, beaucoup de Trinidadiens retrouvent et apprécient des qualités supposées être des aspects clef de leur expérience nationale: créativité locale, lutte, réussite, et festivité » (Stuempfle $1995: 234)^{14}$.

La montée chromatique est destinée à faire l'effet d'une prouesse technique, ce que l'on peut peut-être rapprocher de l'idée de difficulté et de lutte. Elle est en tout cas un passage quasi obligé pour accéder au thème, connu de tous, et au moment fort qui doit éclater à la fin du morceau, où comme le disait Jit Samaroo, arrangeur des Renegades, "You must get high!». Les chromatismes créent une attente, une tension qui amènent un moment de 
cohésion et de joie. Sur les enregistrements en situation, on entend en effet clairement les cris de la foule accompagner ces passages.

Que ces suppositions soient justifiées ou non, l'omniprésence des chromatismes tout au long des arrangements prouve en tout cas qu'il ne s'agit pas seulement d'un outil de séduction des juges. Une conjonction de facteurs favorise leur utilisation. La facilité d'exécution gestuelle y contribue très fortement, en ouvrant des possibilités qui présentent des atouts d'arrangement.

Un autre type de chromatisme pourrait être attribué à la configuration du tenor, sans être pourtant directement lié à la gestuelle. Ce trait musical a la fonction d'amener les changements de tonalité au cours d'un développement. Il s'agit en fait d'une modulation opérée par une série de transpositions du même fragment mélodique en marche d'escalier, par chromatismes. Plutôt que d'intégrer la modulation dans un segment mélodique plus long ${ }^{15}$, les arrangeurs ont en effet l'habitude de répéter le même fragment musical en le transposant successivement dans toutes les tonalités intermédiaires, pour arriver à celle voulue. Le même procédé avait déjà été décrit par Gilbert Rouget à propos de chants rituels béninois : « Le découpage choisi permet d'entendre trois fois l'exposition du chant, c'est-à-dire le déroulement de cette longue descente chromatique au cours de laquelle le même thème est transposé, à chaque énoncé, un peu plus bas. Il est commode et, je crois conforme à la réalité, de parler ici de chromatisme 'transpositeur'. En effet le thème lui-même est diatonique et c'est la manière dont il est transposé légèrement plus bas à chaque énoncé successif qui constitue le chromatisme de cette musique.» (Rouget $1961: 42)$.

Fig. 8 : Transcriptions de chromatismes transpositeurs : extrait de Musical Volcano, par les Desperadoes, 1992, arrangement de Robert Greenidge.

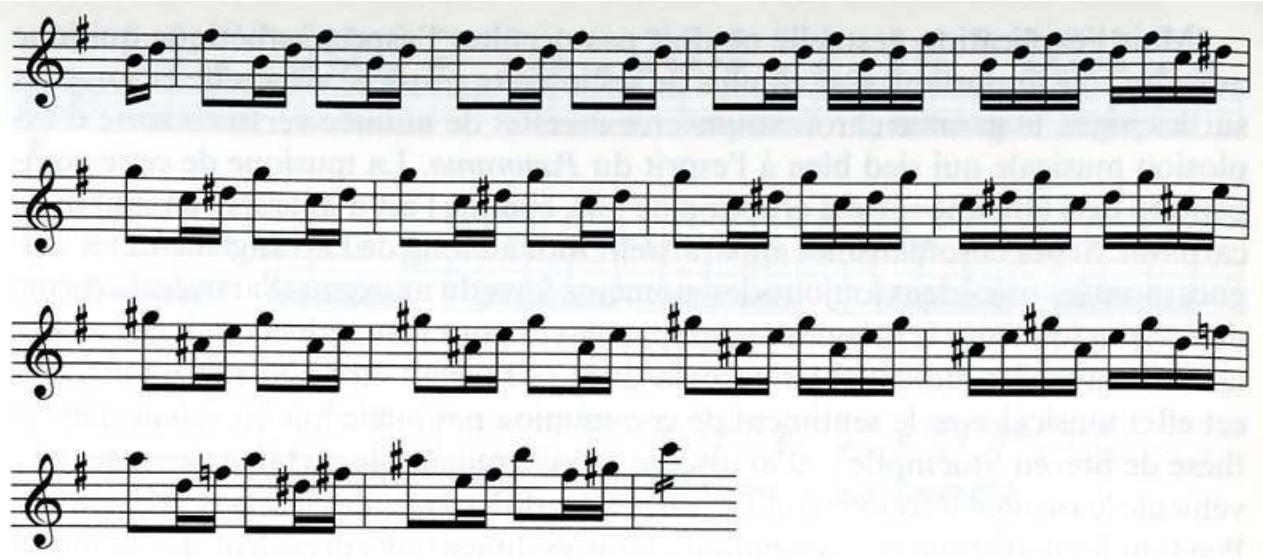

Le chromatisme transpositeur est le moyen le plus courant d'amener une modulation dans les steelbands et, contrairement aux chants décrits par Gilbert Rouget, c'est plutôt dans un mouvement ascendant que s'opère la transposition. Comme on l'a vu précédemment, la topologie du tenor facilite beaucoup la transposition puisqu'il suffit de renouveler le même parcours de notes en le déplaçant par symétrie axiale.

Le geste n'est en revanche pas nécessairement facilité dans la mesure où le déplacement du dessin mélodique implique des changements dans la répartition des frappes entre la main gauche et la main droite. Aucune régularité motrice ne découle donc cette fois du trait musical. Le phénomène de rotation mentale peut en revanche aider à comprendre la 
conception de ce procédé de modulation. Plus que gestuelle, l'influence de l'instrument sur la musique prendrait donc ici la forme d'une inspiration visuelle.

Il apparaît assez clairement que ces préférences gestuelles sont étroitement liées à la rapidité d'exécution des morceaux de compétition et aux difficultés que posent les runs pour la mémoire. Ces démonstrations de virtuosité, où les pannistes peuvent jouer une très longue succession de doubles-croches à un tempo moyen de 130 à la noire, et pouvant aller jusque 150, sont une conséquence directe de la volonté de domination dopée par la compétition. Aucun enchaînement n'est techniquement impossible sur un tenor, pourvu qu'il soit monodique. Mais la complexité de la mémorisation des phrases se répartissant irrégulièrement sur l'instrument à tempos très élevés a orienté la composition des runs vers une recherche de cohérence gestuelle, qui a permis de pousser les limites de la longueur et de la rapidité des phrases en conservant la pratique non professionnelle et occasionnelle de l'instrument ${ }^{16}$.

Geste et mémoire interviennent donc tous deux dans la conception des traits musicaux présentés. Nous allons voir maintenant qu'ils sont également indissociables lors de l'exécution, depuis l'apprentissage jusqu'à la performance.

\section{Geste et apprentissage}

35 Ce qui frappe dans l'apprentissage d'une nouvelle partie musicale, c'est l'importance cruciale dévolue à l'observation gestuelle. L'écoute ne semble pas de prime abord l'élément vraiment essentiel.

Lorsque l'arrangeur veut transmettre une nouvelle partie, il vient lui-même, sans mot dire, la jouer sur un instrument. Il se place à côté d'un musicien spécialement rapide pour la mémorisation, en général chef de section. Ce dernier, ainsi que les pannistes immédiatement voisins s'exercent aussitôt et intègrent la nouveauté avec une rapidité saisissante en observant le parcours des mains de l'arrangeur, tandis que tous les autres membres de la section se pressent autour d'eux en une légère bousculade, pour apercevoir, sur la pointe des pieds, des bribes mélodiques. Ceux qui ont la chance d'être tout près retournent à leur instrument dès qu'ils estiment avoir visualisé la partie, mais peut-être se déplaceront-ils à nouveau pour compléter la fin.

L'arrangeur joue la nouvelle phrase en boucle, jusqu'à ce que l'un de ses voisins immédiats en ait retenu la totalité. Il se retire alors et va tranquillement montrer la partie d'une autre section. Au fur et à mesure que les pannistes assimilent les nouveaux éléments, le groupe se disloque en plusieurs petits attroupements, où les musiciens n'ayant pas encore la totalité de la nouvelle partie observent leurs camarades qui s'entraînent. Chacun s'exerce ensuite un peu dans son coin, mais les pannistes travaillent bientôt tous ensemble en répétant inlassablement en boucle la phrase musicale nouvelle.

Nul besoin d'échange verbal ni de chant, sauf lorsque l'arrangeur n'est pas capable de jouer sur n'importe quel pan; il énonce dans ce cas au chef de section les notes par leur nom ( $A, B, C . .$.$) , et tape le rythme de la mélodie sur le bord de l'instrument, montrant$ quand même certaines notes du doigt ${ }^{17}$. C'est alors autour de son chef que se presse l'ensemble de la section et que se transmet visuellement la nouvelle partie.

On ne peut réduire la mémorisation à un seul phénomène cognitif. Elle est le résultat d'une conjonction d'éléments qui la rendent possible. Mais ce qui frappe d'emblée est l'importance cruciale du facteur visuel. On ne peut naturellement pas en dissocier la 
dimension auditive, nécessairement simultanée, mais on pourra remarquer qu'à elle seule, elle ne suffit pas ${ }^{18}$. «En fait, beaucoup de joueurs sont plus dépendants de leurs yeux que de leurs oreilles pour apprendre la musique - non pas parce qu'ils lisent, mais parce qu'ils regardent sur les autres pour apprendre leur partie ", remarque S. Dudley (1997: 269) ${ }^{19}$ : tous les pannistes commencent effectivement par l'observation avant d'aller s'essayer à jouer.

Lors de l'apprentissage d'un morceau de Panorama dans le steelband Merry Tones, le rôle $\mathrm{du}$ facteur visuel m'apparut un soir clairement à travers l'anecdote suivante. Je m'exerçais alors un peu seule en répétant une nouvelle phrase musicale, quand une jeune fille de ma section vint gentiment jeter un coup d'œil par-dessus mon épaule pour vérifier le bon déroulement de mon apprentissage. Y voyant une erreur, elle la manifesta et, observant plus attentivement, attendit que la boucle musicale en revienne au passage en question. Je suis persuadée qu'en apercevant la faute, elle n'avait pas distinctement entendu ce que j'avais joué : le panyard ${ }^{20}$ était alors plongé dans ce moment de pagaille où, dans une intense cacophonie, chacun s'exerce individuellement pour mémoriser rapidement la nouvelle partie avant de la faire tourner en boucle tous ensemble. L'erreur avait seulement été vue, et était apparue sans aucune hésitation à la jeune fille.

41 Mais au retour du passage incriminé, alors qu'elle s'était rapprochée et écoutait plus attentivement, le doute survint. La note que je jouais ne sonnait pas faux et pouvait parfaitement s'intégrer dans la phrase musicale sans en perturber la logique. «Play it again?" dit-elle à la fois intriguée et dubitative. Ne pouvant trancher à cette nouvelle écoute, elle alla observer un camarade. Elle revint ensuite confirmer et corriger l'erreur.

Ce que suggère cette anecdote, et qui correspond parfaitement aux sensations ressenties lors de l'apprentissage, c'est que l'on reproduit d'abord des données gestuelles, auxquelles les informations auditives parallèles apportent une vérification. Si ce que l'on a photographié produit la même musique que l'on vient d'entendre, l'apprentissage est validé. Ici, mon geste est apparu avec évidence comme faux à cette jeune panniste, qui en douta ensuite par son aspect musicalement plausible. L'observation visuelle apportait une certitude, et l'écoute, le doute. De fait, c'est sa conviction première qui était la bonne.

Le geste acquiert donc ici dès l'apprentissage une place aussi importante que la musique qui en découle. Il est son propre vecteur de transmission, s'enseigne au moins autant par son image que par sa finalité musicale. S'il n'intervient plus ensuite dans l'apprentissage, on va voir qu'il conserve néanmoins, dans sa dimension collective, un rôle important au cours des performances.

\section{Une mémoire contextuelle : la contribution du jeu collectif}

On sait depuis certaines études en psychologie cognitive (Baddeley $1992: 289$ ) que l'on se remémore mieux un phénomène s'il se présente dans le même contexte que lors de l'apprentissage: des plongeurs sous-marins ayant appris une liste de mots en situation immergée et sur la plage, pouvaient significativement mieux se les remémorer si le rappel avait lieu dans la même situation que l'apprentissage. Un « effet net de dépendance » a été mis en valeur, prouvant que le contexte de l'encodage joue sur la mémorisation. Dans les steelbands, il semble effectivement que les musiciens soient très dépendants de l'environnement dans lequel ils apprennent, celui-ci jouant le rôle d'une sorte d'aide 
mémoire. Un changement de contexte peut en effet parfois plonger dans la sensation angoissante de tout oublier.

\section{Guidage gestuel à distance}

Dès l'apprentissage, le panniste, en utilisant fortement des repères visuels, inféode sa mémoire à tout l'environnement, constitué en particulier du jeu très visible des musiciens voisins. La transmission atypique d'une partie de double guitar que j'ai une fois observée illustre bien l'importance mnémonique de la vision des gestes des pannistes du même instrument. La méthode employée semble révélatrice des phénomènes qui entrent en jeu pendant la performance. Cet exemple, toutefois exceptionnel, est dû à la disposition des musiciens ce soir-là dans le panyard de Merry Tones : la musicienne concernée se trouvait isolée des autres membres de sa section. Les instruments étaient répartis à la fois au sol et sur le char, grande plate-forme utilisée pour le défilé du carnaval et par la section rythmique ${ }^{21}$, lors du Panorama.

Une jeune joueuse de double guitar n'avait aucun camarade de section aux alentours, et n'avait pas bien retenu ce qu'elle avait observé sur l'un d'entre eux, quelques instants auparavant. La plupart des autres musiciens connaissaient la nouvelle partie, qui tournait déjà en boucle. L'arrangeur, passant par là, la vit dans l'expectative et entreprit (chose rare) de l'aider. Mais, se trouvant en contrebas du char où elle était installée, dominé de plus d'un mètre, il ne pouvait lui montrer directement la partie comme il l'aurait fait en temps normal. Plutôt que de faire tout un détour pour se placer à côté d'elle, il resta alors en face d'elle et lui mima la gestuelle de sa partie, en miroir, en temps réel.

Ceci n'était rendu possible que par le fait que la fillette jouait des strummings: les gestes de l'arrangeur ne détaillaient pas le rythme à effectuer, qu'elle connaissait déjà. Ses mouvements, assez stylisés, reproduisaient avec les bras la chorégraphie générale de cette succession d'accords comme si, finalement, elle avait eu un autre joueur de double guitar, de dos, devant elle. Comme elle avait déjà regardé plus précisément jouer l'un d'entre eux, elle n'eut aucun mal à retrouver les notes et se remit bientôt à jouer en suivant la chorégraphie reproduite par l'arrangeur.

Cette anecdote est un indice assez révélateur de la manière de mémoriser le répertoire. Le jeu du steeldrum est en effet une chorégraphie qui semble dictée autant par la musique elle même que par le jeu très visuel de mimétisme au sein de la section. Ici, la panniste avait bien quelque part visuellement en mémoire les notes qu'elle avait aperçues chez quelqu'un d'autre. La musique était déjà jouée, la musicienne pouvait donc entendre l'évolution de la grille. Mais pour restituer ces informations qu'elle avait partiellement intégrées, il ne lui manquait qu'une seule chose, la chorégraphie d'un camarade. C'est le jeu collectif qui pouvait, selon l'expression citée par Baddeley (1992: 286), rendre disponible une donnée musicale qui n'est accessible qu' individuellement. 
Fig. 9 : Guidage gestuel à distance.

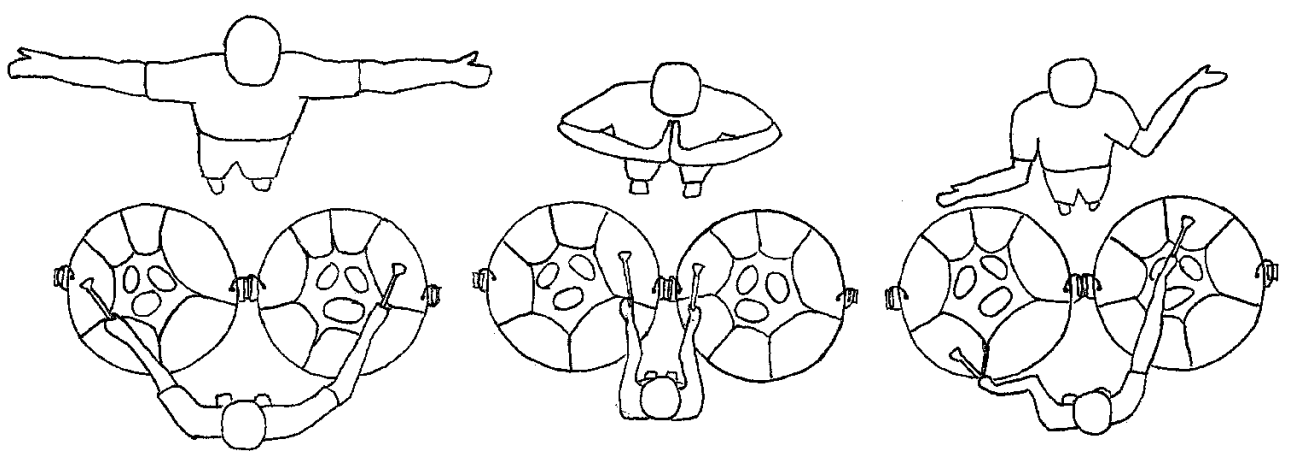

C'est sans doute un phénomène qui contribue fortement à la facilité d'accès de l'instrument. Si le steeldrum peut devenir, à partir d'un certain tempo ou en situation de soliste, d'une complexité technique extrême, c'est en revanche à vitesse lente et au sein d'un groupe un instrument très gratifiant pour les débutants : les hauteurs de notes ne posent pas, comme au violon, de grosses difficultés d'ajustement, et la mémoire de la pièce peut se faire même si le novice ne connaît pas encore la répartition des notes sur son instrument, grâce à la mémoire visuelle et au renfort de la mémoire des voisins, surtout s'ils sont plus confirmés. La fillette que nous avons mentionnée ci-dessus ne jouait ainsi du double guitar que depuis quelques mois, et participait pour la première fois au Panorama.

\section{Mimétisme de section}

Mais l'influence du jeu collectif sur la mémoire n'est pas l'apanage des débutants, même si c'est peut-être plus flagrant chez eux. Elle concerne aussi les pannistes les plus experts, et pas seulement en condition d'apprentissage d'une nouvelle pièce mais en toute situation de jeu. Il est ainsi frappant de constater que les pannistes ont énormément de mal à jouer seuls. Il m'est en effet arrivé de demander à des membres de chaque section de jouer individuellement, simplement accompagnés de quelqu'un frappant la pulsation, afin d'enregistrer et de transcrire plus facilement les différentes voix du morceau. J'ai d'abord été confrontée au refus de plusieurs d'entre eux pourtant habituellement coopérants, qui répondaient tout gênés : « Mais je n'y arriverai pas!», «Je ne m'en souviens plus!», ou « Demande plutôt à Untel... ».

Ces hésitations venaient visiblement du fait que les priver du reste de l'orchestre les plaçait dans une situation trop inconfortable. Les musiciens qui acceptèrent éprouvèrent effectivement des difficultés, même les plus confirmés. Les joueurs qui s'étaient prêtés au jeu, hormis un jeune joueur de double guitar qui - ayant assez peu d'années de pratique eut vraiment un mal fou à arriver au bout, étaient reconnus comme parmi les meilleurs. Or à l'exception d'un bassiste (du steelband Merry Tones), tous s'interrompirent au moins une fois. Pour limiter la mise en danger, certains demandèrent à un camarade de section de jouer avec eux. Malgré cela, deux bassistes des Renegades, groupe fort réputé et vainqueur de neuf Panoramas, s'interrompirent, exactement en même temps. Un joueur de double guitar des Renegades, faisant partie du stage side, ayant donc toute l'année une pratique quasi quotidienne de son instrument et ayant participé à plusieurs tournées mondiales, me dit, très vexé d'avoir dû s'y reprendre à trois ou quatre fois : «Dis is de 
woooorse time I ever played dis tune! $\aleph^{22}$. On était la veille de la finale du Panorama; il était donc dans les conditions d'entraînement optimum.

Ces constatations n'enlèvent évidemment rien aux excellentes qualités de tous ces musiciens, qui sont capables de réaliser des prouesses techniques à une vitesse stupéfiante. Elles montrent cependant que, accoutumée à un certain contexte, leur mémoire est étroitement dépendante de celui-ci. Le trac peut bien sûr expliquer en partie leur hésitation. La présence d'un micro est évidemment troublante. Ces remarques proviennent de situations qui n'étaient de toutes façons pas rigoureusement expérimentales. Mais le phénomène semble trop récurrent pour qu'on puisse entièrement l'attribuer à une timidité passagère.

ensations ressenties en jouant ainsi collectivement du steeldrum vont d'ailleurs tout à fait dans ce sens. Sans même regarder fixement ses voisins, leurs gestes, très amples en raison de la taille de l'instrument, entrent naturellement dans le champ de vision du panniste. Les mouvements sont en effet très visibles puisqu'ils impliquent au minimum le poignet, souvent tout le bras, le buste ou parfois même, pour les basses, le corps tout entier. La communion gestuelle avec les autres membres de la section, emportée comme un seul corps dans un mouvement unique, maintient le musicien dans une vérification simultanée de ses propres réflexes.

Lorsque ce système de repères est perturbé, comme dans le cas du jeu solitaire, des erreurs surviennent. En groupe, en cas de trou de mémoire, une observation anticipée d'un voisin de section peut permettre d'interroger son intention gestuelle afin d'accéder à une remémoration correcte. L'effet de communion est assez fort pour que l'on puisse deviner le mouvement de ses voisins avant même qu'il ne soit engagé. Si un joueur voit qu'il va faire une fausse note, il peut dans le meilleur des cas avoir encore le temps de la corriger : l'amplitude des gestes permet parfois de compenser un mauvais réflexe par une forte accélération de dernière minute.

Cela peut bien sûr avoir des effets pervers : il est possible que, dans un excès de confiance, on soit emporté dans la chorégraphie du voisin et que l'on joue exactement la même fausse note en même temps. L'erreur d'un voisin peut semer le doute et être contagieuse. Une information contraire à son intuition gestuelle est très troublante et peut avoir un effet paralysant. Ce n'est pas un hasard, par exemple, si les deux bassistes des Renegades se sont arrêtés précisément au même instant, sur les dix minutes que dure le morceau. Le jeu du steeldrum relève d'un subtil équilibre entre les réflexes individuels et la confiance que l'on porte à son entourage.

Mais, en dépit de ces situations occasionnellement perturbantes, cette caractéristique de la mémoire a un effet général très rassurant. La section entière semble animée d'un même mouvement, dont les musiciens n'ont plus qu'à suivre la chorégraphie en toute confiance, sans réfléchir. Le sentiment de communion généralement recherché en musique est ici ressenti très fortement, puisqu'en plus de la synchronie musicale, il y a une coïncidence chorégraphique spectaculaire. Le panniste a sa propre connaissance du morceau, et perçoit simultanément avec une grande sensibilité les intentions gestuelles voisines, générant cet état de mimétisme de section.

\section{Un ballet musiquant}

Un mode d'apprentissage très visuel, une vérification systématique des réflexes gestuels par l'observation de la synchronie du groupe, un ensemble de personnes se mouvant 
comme un seul corps... Le jeu du steeldrum comporte bien des points de ressemblance avec la danse collective. Si la chorégraphie est largement soumise à la mélodie de la pièce et au dessin qu'elle forme sur les instruments, c'est cependant des mouvements dansés que l'on voit dans les gestes des pannistes. Dans sa réflexion sur l'origine des instruments de musique, André Schaeffner a déjà bien mis en évidence les liens entre la musique instrumentale - notamment percussive - et la danse, en montrant leur origine commune :

« 'On se demandera si le plaisir de battre un tambour est né du besoin, non seulement de marquer le rythme, mais de participer encore de quelques manière à la gesticulation générale. Taper sur le premier objet venu, chaudron, pot de terre ou tronc d'arbre, c'est répondre tout autant à un réflexe de danseur qu'à un geste d'instrumentiste' ${ }^{23}$. C'est ainsi que bien des maniements d'instruments sonores, par leur violence ou même par leur seul aspect rythmique, ressortissent encore de la danse, tout comme les sauts et les piétinements dont nous étions partis. [...] Là même où elle gouverne tout geste, la musique n'en forme pas moins un spectacle, bruissant, et qui modifie nos idées modernes ou 'classiques' - sur le ballet. Toutes relations entre musique et danse comportent quelque artifice, hors celles qui mêlent étroitement gestes de danseurs et gestes d'instrumentistes » (Schaeffner 1968 : 38-39).

Fig. 10a : Panorama 1998 : section de tenors de Tokyo.

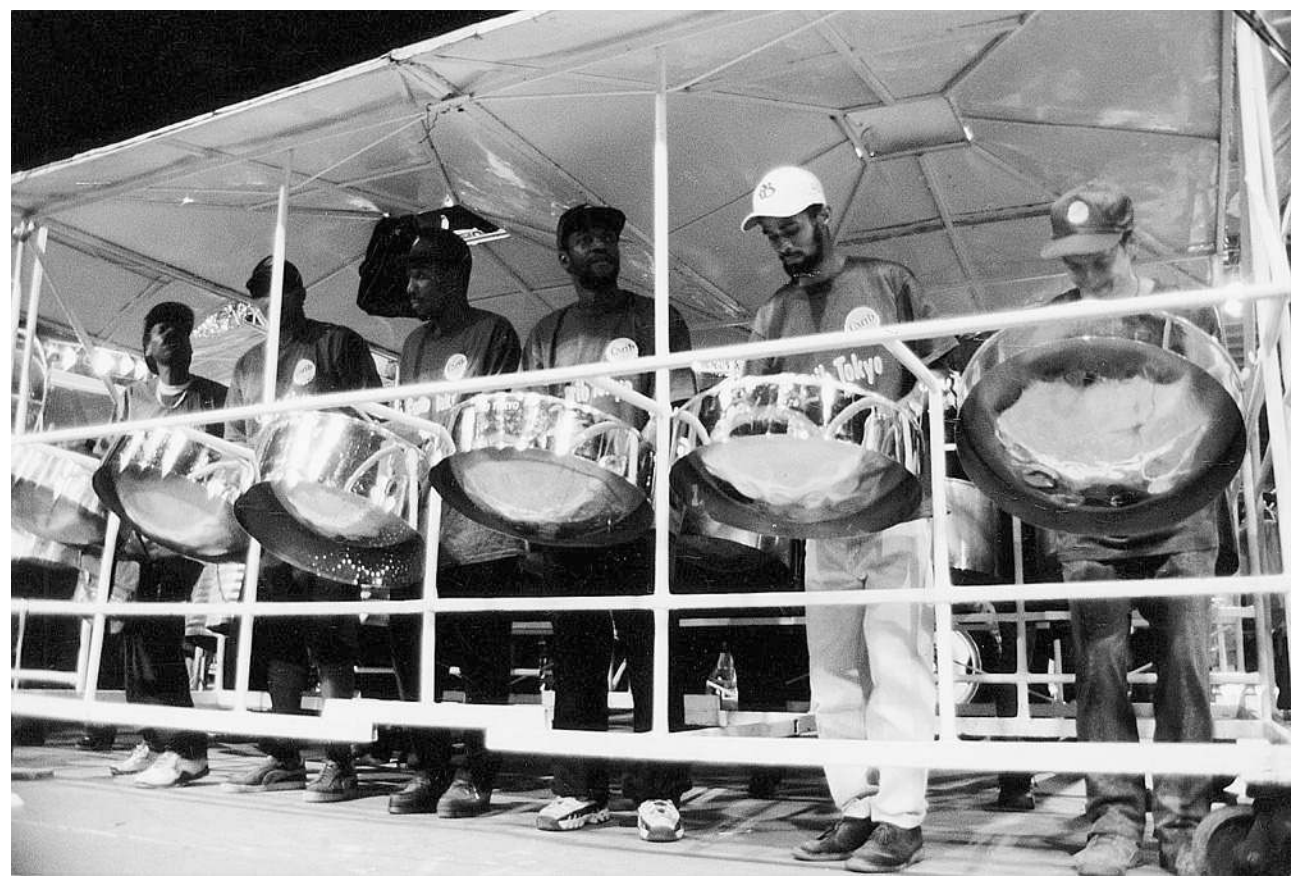


Fig. 10b : World Pan Festival 2000 : section de basses des Court Laventille Sound Specialists.

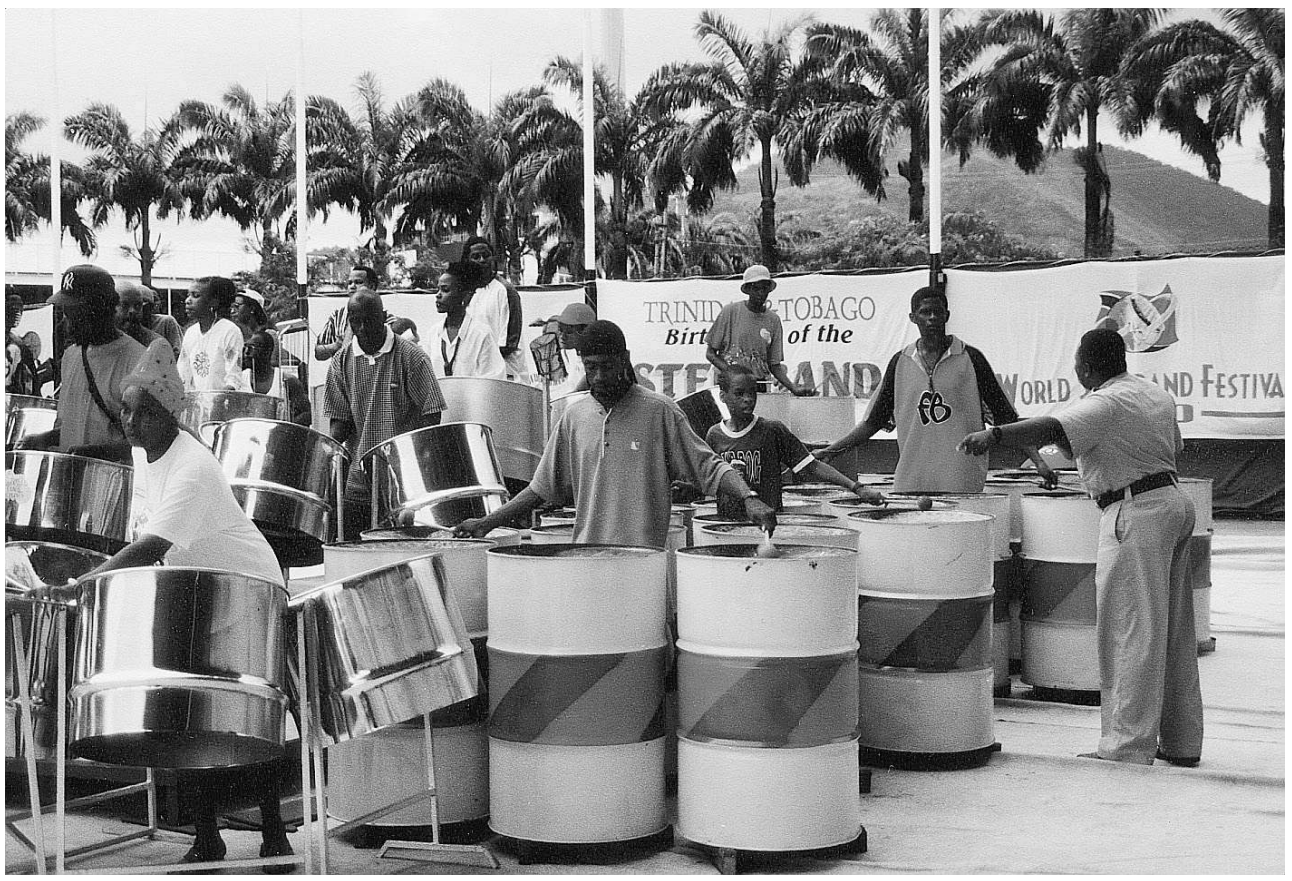

59 Un steelband offre en effet le spectacle fascinant d'une sorte de ballet musiquant. Les différentes sections ont de larges mouvements rythmés et synchronisés. La musique des steelbands perd par conséquent beaucoup à l'enregistrement. Non seulement le son puissant et envoûtant de la centaine de musiciens est terriblement réduit, mais toute la dimension spectaculaire est amputée. Les radios trinidadiennes ne diffusent d'ailleurs que tout-à-fait exceptionnellement des pièces de steelbands, en dehors de la transmission des compétitions. La population se déplace volontiers au panyard pour assister à la répétition du steelband du quartier, va vivre la liesse du Panorama ou le suit à la télévision, et s'arrêtera avec plaisir lorsqu'un groupe joue au détour d'une rue pour une quelconque manifestation publique. Mais la musique des steelbands ne fait pas vraiment partie de ce que l'on entend diffusé à longueur de journées dans les rues de Port-of-Spain, provenant de lieux privés ou publics. Les personnes réellement intéressées par les enregistrements sont très souvent elles-mêmes pannistes, ou alors de fervents supporters.

Il est en effet franchement dommage de ne pas voir un steelband en action. Même pour une pièce classique européenne lors du Festival, où la sobriété est de rigueur, l'ensemble du groupe se meut avec délicatesse et marque la pulsation par un balancement régulier du buste. Au Panorama, en plus de la vitesse et de l'aspect plus rythmique de la musique (et donc de la chorégraphie), c'est la surenchère d'exubérance. On balance son buste, bien sûr, mais on tape des pieds, on saute, on pirouette dès que l'occasion se présente. Le public se souviendra de ce joueur de double guitar qui, tel un musicien de hard rock, s'est jeté à genoux tout en continuant à jouer en secouant ses ras $^{24}$, ou de ce bassiste profitant d'un break pour jaillir hors de son instrument et exécuter quelques déhanchements sous les ovations générales. La musique crée un canevas chorégraphique sur lequel toutes les initiatives personnelles sont bienvenues. 


\section{Conclusion}

61 La musique, comme l'essentiel des créations humaines, relève de l'action médiate. Il n'est de pensée pure, et on sait depuis le travail de Jack Goody (1979), que la forme du moyen d'expression oriente le résultat de la production. Il a ainsi montré que l'écriture a modifié profondément les modes de réflexion de tradition orale, et que l'émergence de la pensée scientifique moderne est par exemple l'héritière logique de son invention.

De même que l'écriture a permis la forme romanesque, le geste permet la musique à plusieurs titres. En tant qu'acteur, bien sûr, mais les recherches de John Baily $(1977,1987)$ ont aussi montré qu'il n'est pas simplement un neutre exécutant de décisions abstraites. Comme le suggère à nouveau ce travail, le mode "spatio-moteur " de l'exécution musicale peut avoir sa part de créativité. Le geste a sans doute permis certaines idées musicales. S'il influence la composition, on a vu qu'il doit tout de même présenter des atouts esthétiques pour être couramment utilisé. C'est un faisceau d'avantages qui crée la récurrence de traits comme les chromatismes dans les steelbands de Trinidad. Le geste permet également la musique dans la mesure où l'amplitude du premier favorise le stockage mnésique de la seconde en contexte collectif, donnant lieu à des pièces longues, rapides et complexes.

Des chercheurs (Stuempfle 1995, Dudley 1997) ont déjà montré le rôle de cohésion nationale assumée par le steeldrum : «Le pan, en tant qu'instrument festif indigène, est un moyen vital par lequel la nation se crée, se contemple, et se célèbre elle-même » (Stuempfle 1995: 236) ${ }^{25}$. Il semblerait qu'en plus du contenu symbolique fortement identitaire de l'instrument, les steelbands, par leur jeu dansé et la mémorisation en quelque sorte partagée dans le groupe, rempliraient particulièrement bien leur rôle de fédérateurs sociaux.

\section{BIBLIOGRAPHIE}

AHO Wiliam R., 1987, « Steel Band Music in Trinidad and Tobago: The Creation of a People's Music ». Latin American Music Review VIII/1. University of Texas Press: 26-56.

BADDELEY Alan, 1992, La mémoire humaine. Paris : PUG.

BAILY John, 1977, « Movement pattern in playing the herati dutar », in John Blacking ed.: The Anthropology of the Body. London, New-York, San Fransisco : Academic Press : 275-330., 1987, « Principes d'improvisation rythmique dans le jeu du rubâb d'Afghanistan », in Bernard LortatJacob, dir. : L'improvisation dans les musiques de tradition orale. Paris : SELAF :177-188.

BRIL Blandine, 1991, " Les gestes de percussion : analyse d'un mouvement technique ", in Denis Chevallier, dir. : Savoir faire et pouvoir transmettre. Collection Ethnologie de la France 6. Editions de la Maison des Sciences de l'Homme : 61-80.

COWLEY John, 1996, Carnival, Canboulay and Calypso; Traditions in the making. Cambridge: Cambridge University Press. 
DUDLEY Shannon, 1997, Making music for the nation: Competing Identities and Esthetics in Trinidad and Tobago's Panorama Steelband Competition. Ph.D dissertation. Berkeley: University of California Berkley.

GOODY Jack, 1979, La raison graphique. La domestication de la pensée sauvage. Paris : Éditions de Minuit.

ROUGET Gilbert, 1961, « Un chromatisme africain », L’Homme (Paris) I, 3 : 32-46.

SCHAEFFNER André, 1968, Origine des instruments de musique. Paris : Mouton / Maison des Sciences de l'Homme.

STUEMPFLE Steven, 1995, The steelband movement. The forging of a national art in Trinidad and Tobago. Philadelphia : University of Pennsylvania Press.

\section{NOTES}

1. «Casserole » en anglais. Ce terme est synonyme de steeldrum et steelpan, à ceci près qu'il désigne parfois plus précisément un seul des bidons de l'instrument. Un steeldrum peut donc être constitué de plusieurs pans.

2. Ce concept emprunté à la psychologie (qui l'a elle-même emprunté à la rhétorique), désigne ici le détournement d'un objet de sa fonction initiale.

3. La population de Trinidad \& Tobago a des origines multiples. L'Encyclopaedia Universalis évalue la communauté d'origine indienne à $40,3 \%$, l'africaine à $39,6 \%$, la métisse à $18,4 \%$, l'européenne à $0,6 \%$, la chinoise à $0,4 \%$, et les autre à $0,7 \%$ (chiffres de 1990).

4. Ce travail repose d'une part sur mon expérience de panniste et sur un terrain de deux mois lors duquel je me suis intégrée à un steelband pour le Panorama. La première partie de l'article résume mon travail de maîtrise et la seconde touche à mon sujet de thèse, dont cet article ne saurait être le strict résumé : des expérimentations sur le modèle des sciences cognitives viendront mesurer finement les caractéristiques de ce type de mémoire, permettant une analyse plus complète par une dissociation des niveaux de musiciens et des différents paramètres de l'encodage.

5. A titre d'exemple, en 2001, 57 conventional bands (comprenant jusque 100 musiciens) 51 traditional bands (jusque 45 musiciens) ont concouru, auxquels il faut ajouter 23 groupes juniors (source internet, site de Pan Trinbago). Les conventional bands ont des instruments pouvant être composés de plusieurs pans pour un seul musicien. Les traditional bands portent leurs instruments en bandoulière, chaque panniste n'ayant donc qu'un bidon.

6. Du français « ramager».

7. Joueur de steeldrum. Ce terme neutre d'instrumentiste possède d'après Dudley (1997: 17) une nuance avec le terme panman pouvant parfois avoir en sus une connotation de lutte sociopolitique liée à l'histoire de l'instrument, absente du contenu sémantique de « pannist » (francisé ici en panniste).

8. L'axe pris en compte se réfère au diamètre supérieur du cylindre, pris dans l'axe de l'instrumentiste.

9. Nous appellerons «cellule » les groupements de 2 à 4 doubles-croches, c'est-à-dire des unités de répétitions de un temps ou moins.

10. "And sometimes it's not being practiced to rhythm at all, because the piece is very intricate with a lot of notes in the runs, and you only hearing bdddddup, bdddddup! (imitating the sound of a rapid chromatic run) for about an hour ".

11. "If I'm a tourist or whatever and I come to Panorama and I listen for the first 5 bands and realize that all I'm hearing is chromatics, useless chromatics, I will go home» (ibid.: 214). [...] « For instance if you 
notice, all, majority of the bands, $90 \%$ of the bands play chromatics. Most of the composers, when you compose a tune, you don't just put in chromatics because the other composer did it. You arrange your tune to the ability you have and you know what you want to hear. You must arrange your tune for yourself and for the players, not for the judges. Because the judges want to hear chromatics. You cannot and should not put in chromatics because they say that that is the only way to build excitement- that is not the only way to build excitement ».

12. On constate l'alternance droite/ gauche, les seuls doublés étant gauche-gauche entre le fa et le fa\# ou droite-droite entre le fa\# et le sol, et droite-droite entre le si et le do.

13. "Pan is easy... if you practice chromatics".

14. "Earlier in the chapter I discussed certain messages that the steelband has carried concerning ethnic and class differentiation and integration. At performance occasions, however, other dimension prevail. In listening to bands strive to execute complex musical arrangements without flaw, many Trinidadians reencounter and appreciate qualities that are believed to be key aspects of their national experience: local creativity, struggle and achievement, and festivity " (Stuempfle 1995: 234).

15. Certains arrangeurs comme Jit Samaroo, plus profondément influencés par la musique classique occidentale, utilisent plutôt des modulations de ce type, ce qui est toutefois rare.

16. La majorité des pannistes ne joue que lors du Panorama : environ un mois et demi par an de répétitions quotidiennes avant les soirs de performance.

17. Dans les steelbands que j'ai observés (Merry Tones et Renegades), les arrangeurs savent jouer de tous les types de steeldrum. Cette information provient du film de Verba et Mrejen, Pan in A Minor, et est confirmée par les témoignages de Guillaume Kervel et Mathieu Borgne, pannistes français ayant participé à plusieurs Panoramas.

18. Il existe bien entendu des pannistes aveugles ou malvoyants. Leur handicap ne leur interdit évidemment pas la pratique du steeldrum ; un sens se développe toujours pour remplacer celui qui est défectueux. L'apprentissage d'oreille est tout à fait possible, mais il est alors choisi par défaut. 19. "In fact, many players are more dependant on their eyes than their ears for learning music - not because they read, but because they watch on others to learn their parts ".

20. Lieu de répétition du steelband, comprenant généralement un bâtiment couvert et une cour extérieure.

21. La section rythmique, appelée aussi engine room, comporte au Panorama une vingtaine de musiciens. Elle est constituée d'une batterie, de congas (utilisés par paires, et joués avec des baguettes de steeldrum), de cloches en plastique, et surtout d'idiophones métalliques d'une terrible puissance sonore: le scratch, sorte de râpe de cuisine cylindrique, est frotté avec un peigne en fer, et l'iron, tambour de frein de camion, martelé avec des clous. D'autres types d'instruments s'intègrent à cette section selon les choix d'arrangement.

22. «Je n'ai jamais joué ce morceau aussi mal! ».

23. Auto-citation : Schaeffner, Notes sur la musique des afro-américains, p. 297.

24. On appelle ras à Trinidad les dreadlocks («terribles mèches »), coiffure des rastas de Jamaïque. 25. "The pan, as an indigenous instrument of festivity, is one vital means by which the nation creates, contemplates, and celebrates itself $"$. 


\section{RÉSUMÉS}

Depuis l'indépendance à Trinidad \& Tobago, le steeldrum représente un véritable symbole national, où des compétitions annuelles absorbent l'essentiel de l'énergie musicale. La plus énorme, le Panorama, mobilise des steelbands de cent personnes. Le geste est abordé dans cet article comme l'un des constituants actifs de la performance, d'abord dans la composition de certains traits musicaux et ensuite dans les procédés mnésiques qu'il implique en contexte collectif. Dans les runs (longue succession de double-croches) de tenor, principal instrument mélodique, on remarque la récurrence de phrases musicales impliquant une régularité des frappes entre la main gauche et la main droite, recherche d'un équilibre moteur soulageant la mémoire. En groupe, l'effort de mémoire est aussi aidé par la gestuelle des compagnons de jeu qui entre de fait dans le champ visuel du musicien, chorégraphie générale le maintenant dans la vérification simultanée de ses propres réflexes mnésiques.

\section{AUTEUR}

\section{AURÉLIE HELMLINGER}

Aurélie HELMLINGER, née en 1975, est doctorante en ethnomusicologie à l'université Paris X Nanterre sous la direction d'Aurore Monod-Becquelin, et rattachée au laboratoire d'ethnomusicologie du Musée de l'Homme. Après une licence de berbère (touareg) aux Langues'O, elle se consacre depuis sa maîtrise d'ethnomusicologie à l'étude des steelbands de Trinidad. Ayant pratiqué les polyrythmies mandingues et brésiliennes, elle développe particulièrement depuis fin 1996 la pratique du steeldrum (tenor et double tenor). Elle a joué dans des groupes français et trinidadien, participé au Panorama et au World Pan Festival à Trinidad et joue actuellement dans le steelband Pan à Paname. 